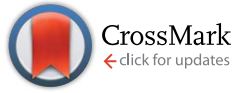

Cite this: RSC Adv., 2017, 7, 12974

DOI: $10.1039 / c 7 r a 90025 b$

www.rsc.org/advances

\section{Correction: Probing the nano-scale architecture of diamond-patterned electrospun fibre mats by synchrotron small angle X-ray scattering}

Tan Sui, ${ }^{a}$ Kirill Titov, ${ }^{\mathrm{b}}$ Siqi Ying, ${ }^{\mathrm{a}}$ Hongjia Zhang, ${ }^{\mathrm{a}}$ Igor P. Dolbnya, ${ }^{\mathrm{c}}$ Jin-Chong $\operatorname{Tan}^{\mathrm{b}}$ and Alexander M. Korsunsky ${ }^{* a}$

Correction for 'Probing the nano-scale architecture of diamond-patterned electrospun fibre mats by synchrotron small angle X-ray scattering' by Tan Sui et al., RSC Adv., 2017, 7, 8200-8204.

In the original manuscript, some relevant funding information was omitted from the Acknowledgements section. The corrected Acknowledgements are given below.

\title{
Acknowledgements
}

Funding for the MBLEM laboratory at Oxford received under EU FP7 project iSTRESS (604646) is acknowledged, along with the access to the facilities at the Research Complex at Harwell (RCaH), under the Multi-disciplinary Centre for In situ Processing Studies (CIPS) supported by EPSRC RCUK (EP/I020691/1), and (EP/P005381/1) Tackling human dental caries by multi-modal correlative microscopy and multi-physics modelling.

Additionally, some information regarding access to supplementary data for the manuscript was omitted. This information is given below.

Additional data can be accessed via ORA (http://ora.ouls.ox.ac.uk). Request for any material samples or specimens described in this manuscript should be directed to the corresponding author.

The Royal Society of Chemistry apologises for these errors and any consequent inconvenience to authors and readers. 